\title{
ONE-PARAMETER TRANSFORMATION GROUPS OF THE THREE-SPHERE
}

\author{
ROBB JACOBY
}

Introduction. In [3], Seifert describes all the fiberings of the three-sphere. The fibers are the orbits when the circle group acts effectively on the three-sphere without fixed points in a certain way. In $[2, \S 6.7]$, Montgomery and Zippin show that when the circle group acts on the three-sphere effectively but with fixed points its action is described in a suitable coordinate system as a rotation about an axis. In this paper the methods of [2] and of [3] are combined to show that the circle group can act effectively on the three-sphere without fixed points in no other way than in one of the ways described in [3]. The result is stated precisely in the following theorem.

The author has had the benefit of frequent conversations with Deane Montgomery during the preparation of this paper.

Notation. In the following pages ' $R$ ' denotes the set of all real numbers, ' $S$ ' denotes the set of all triples $x=\left(x_{1}, x_{2}, x_{3}\right)$ of real numbers satisfying $x_{1}^{2}+x_{2}^{2}+x_{3}^{2}=1$, and ' $S$ ' or ' $S$ ' denotes the set of all quadruples $x=\left(x_{1}, x_{2}, x_{3}, x_{4}\right)$ of real numbers satisfying $x_{1}^{2}+x_{2}^{2}+x_{3}^{2}$ $+x_{4}^{2}=1 . \Lambda$ is the null set.

THEOREM. If $T$ is a continuous function from $R \times S$ to $S$ subject to the conditions:

(a) for arbitrary $t_{1}$ and $t_{2}$ in $R$ and $x$ in $S, T\left(t_{1}+t_{2}, x\right)=T\left(t_{1}, T\left(t_{2}, x\right)\right)$,

(b) $T(1, x)=x$ for arbitrary $x$ in $S$; conversely, there is no $t$ strictly between 0 and 1 for which $T(t, x)=x$ for all $x$ in $S$,

(c) there is no $x$ in $S$ for which $T(t, x)=x$ for all $t$ in $R$, then there are two uniquely determined relatively prime integers $k^{\prime}$ and $k^{\prime \prime}$, with $1 \leqq k^{\prime} \leqq k^{\prime \prime}$, and a homeomorphism $\phi$ of $S$ onto $S$ so that $T(t, \phi x)$ $=\phi\left(\Pi_{\left(k^{\prime}, k^{\prime \prime}\right)}(t, x)\right)$ for all $t$ in $R$ and $x$ in $S$, where $\Pi_{\left(k^{\prime}, k^{\prime \prime}\right)}$ is defined from $R \times S$ to $S$ by

$$
\begin{aligned}
& \begin{aligned}
\Pi_{\left(k^{\prime}, k^{\prime \prime}\right)}(t, x) & =\Pi_{\left(k^{\prime}, k^{\prime \prime}\right)}\left(t,\left(x_{1}, x_{2}, x_{3}, x_{4}\right)\right) \\
& =\left(x_{1} \cos 2 \pi k^{\prime} t+x_{2} \sin 2 \pi k^{\prime} t,-x_{1} \sin 2 \pi k^{\prime} t+x_{2} \cos 2 \pi k^{\prime} t,\right.
\end{aligned} \\
& \left.x_{3} \cos 2 \pi k^{\prime \prime} t+x_{4} \sin 2 \pi k^{\prime \prime} t,-x_{3} \sin 2 \pi k^{\prime \prime} t+x_{4} \cos 2 \pi k^{\prime \prime} t\right)
\end{aligned}
$$

Received by the editors March 18, 1955. 
Preliminary Remarks. The orbit space $S^{*}$ of $S$ under $T$ is the space whose points are the orbits of $T$ and whose topology is the image under $\Phi$, the natural projection of $S$ onto $S^{*}$, of the topology of $S$. Thus $\Phi$ is a continuous, open mapping. Furthermore, $S^{*}$ is metrizable. It is shown in Lemma 2 that $S^{*}$ is homeomorphic to $S^{2}$.

The period function $p$ is defined from $S$ to $R$ by the condition that, for any $x$ in $S, p x$ is the least positive real number for which $T(p x, x)$ $=x$. This is a valid definition since $T$ has no fixed points. It is not hard to see that $p$ assumes rational values which are reciprocals of integers and is lower-semicontinuous. Since $p$ is constant on an orbit it induces a function $p^{*}$ on $S^{*}$ satisfying $p^{*}(\Phi x)=p x$, for all $x$ in $S$. The set $C$ of points of $S$ where $p$ is continuous is open since $p$ is discrete-valued, and is everywhere dense since $p$ is semicontinuous. Orbits in $C$ are called 'ordinary'. The complement $D$ of $C$ in $S$ is closed. Orbits in $D$ are called 'exceptional'.

As is true for the case treated in [2], the inverse $S$ under $\Phi$ of a simple arc, a simple closed curve, and a two-cell in $\Phi C=C^{*}$ are a cylinder, a torus, and a solid torus, respectively. Each of these inverses has a cross-section and is homeomorphic to the topological product of such a cross-section and a simple closed curve. If $A$ is a simple arc in $S^{*}$ with endpoints $a$ and $b$ and if $A-\{b\}$ is in $C^{*}$ whereas $b$ is in $\Phi D=D^{*}$, then $k \cdot p^{*} b=p^{*} x$ for some integer $k$ greater than 1 and all $x$ in $A-\{b\} . \Phi^{-1} A$ is then a twisted strip $B_{k}$ and has a cross-section.

LEMma 1. $D^{*}$ is finite.

Proof. Since $D^{*}$ is closed in $S^{*}$, there exist sets $D_{1}^{*}$ and $D_{2}^{*}$ so that $D^{*}=D_{1}^{*} \cup D_{2}^{*}, D_{1}^{*} \cap D_{2}^{*}=\Lambda, D_{1}^{*}$ is perfect, $D_{2}^{*}$ is countable. The proof in [2] shows that $D_{1}^{*}=\Lambda$. Thus $D^{*}$ is closed and countable.

If $D^{*}$ is infinite a contradiction will be reached. For then $D^{*}$ must contain infinitely many isolated points. Thus, since $D^{*}$ is compact there is a convergent sequence $\left\{X_{n}\right\}$ of distinct isolated points of $D^{*}$. It will now be shown that no two points of this sequence have the same period. Corresponding to any two distinct points $X_{m}$ and $X_{n}$ of the sequence, two points $x_{m}$ and $x_{n}$ in $S$ are chosen with $\Phi\left(x_{m}\right)=X_{m}$ and $\Phi\left(x_{n}\right)=X_{n}$. Since the closed set $\Phi^{-1}\left(D^{*}-\left\{X_{m}, X_{n}\right\}\right)$ is a countable union of closed one-dimensional sets, it is one-dimensional, and so $x_{m}$ and $x_{n}$ can be joined by a simple arc $A_{m, n}$ missing $\Phi^{-1}\left(D^{*}-\left\{X_{m}, X_{n}\right\}\right)$. The image $\Phi\left(A_{m, n}\right)$ is a connected locallyconnected compact subset of $S^{*}$ containing $X_{m}$ and $X_{n}$ and missing $D^{*}-\left\{X_{m}, X_{n}\right\}$; consequently, $X_{m}$ and $X_{n}$ can be joined by a simple arc $C_{m, n}$ missing $D^{*}-\left\{X_{m}, X_{n}\right\}$. If $p^{*}\left(X_{m}\right)=p^{*}\left(X_{n}\right)=k, \Phi^{-1}\left(C_{m, n}\right)$ is obtained by joining two twisted strips $B_{k}$. (Since $p^{*}$ is continuous 
at any $X$ for which $p^{*} X=1$, it is seen that $k>1$.) The second homology group thereof, with integers modulo $k$ as coefficients, is a cyclic group of order $k$, whereas with integral coefficients this group is zero. This is impossible, in view of the Alexander duality theorem. The assumption $p^{*}\left(X_{m}\right)=p^{*}\left(X_{n}\right)$ has thus led to a contradiction.

Since no two terms of the sequence $p^{*}\left(X_{n}\right)$ are equal and since these numbers are reciprocals of integers, it is seen that the sequence converges to zero. Thus a convergent sequence $\left\{y_{m}\right\}$ of points in $S$ can be constructed with $\left\{p\left(y_{m}\right)\right\}$ converging to zero. This is impossible since $p$ is lower-semicontinuous and no point has period zero. The desired contradiction has been reached. $D^{*}$ is finite.

REMARK. $C$ is the complement of a finite number of orbits and so is an everywhere dense connected open set. $p$ then has a constant value, say $a$, on $C$. Since $a$ is an integral multiple of the period along any exceptional orbit, it is seen that $a=1$.

Lemma 2. $S^{*}$ is homeomorphic to $S^{2}$.

PROOF. The proof consists in verifying the characteristic properties of the two-sphere, as given in [4].

Since $S^{*}$ is a continuous image of the three-sphere, it is a locallyconnected continuum.

(a) Let $a$ and $b$ be any two points of $S$ on distinct ordinary orbits. Since $a$ and $b$ can be joined by a simple arc not intersecting an exceptional orbit, it is seen, as in the preceding proof, that the images $\Phi a$ and $\Phi b$ can be joined by a simple arc $A$ in $S^{*}$. Let $P^{\prime}$ and $P^{\prime \prime}$ be any two points of $A$, distinct from $\Phi a$ and $\Phi b$, in the order $\Phi a, P^{\prime}, P^{\prime \prime}$, $\Phi b$, and let $a^{\prime}$ and $b^{\prime}$ be chosen in $S$ so that $\Phi a^{\prime}=P^{\prime}$ and $\Phi b^{\prime}=P^{\prime \prime}$. Let $A^{\prime}$ be the subarc of $A$ from $P^{\prime}$ to $P^{\prime \prime}$. Then $\Phi^{-1} A^{\prime}$ is a topological cylinder in $S$ not containing $a$ or $b$, so $a$ and $b$ can be joined by an arc $\Gamma$ in $S$ having no points in common with $\Phi^{-1} A^{\prime}$. Clearly there are two arcs $\Gamma^{\prime}$ and $\Gamma^{\prime \prime}$ in $\Phi^{-1} A$ joining $a$ to $a^{\prime}$ and $b$ to $b^{\prime}$, respectively, and having only $a^{\prime}$ and $b^{\prime}$ in $\Phi^{-1} A^{\prime}$. The locally-connected continuum $\Gamma^{\prime} \cup \Gamma \cup \Gamma^{\prime \prime}$ gives rise to a simple arc $A^{\prime \prime}$ in $S^{*}$ joining $P^{\prime}$ to $P^{\prime \prime}$ and having no points in common with $A^{\prime}$ except $P^{\prime}$ and $P^{\prime \prime}$. Then $A^{\prime} \cup A^{\prime \prime}$ is a simple closed curve in $S^{*}$.

(b) The inverse $\Phi^{-1} J$ of any simple closed curve $J$ in $S^{*}$ is the union, in a simple way, of a finite number of twisted strips. It is thus seen that $\Phi^{-1} J$ is a two-cycle, with integers modulo 2 as coefficients, and so this inverse separates $S$. This implies that $J$ separates $S^{*}$.

(c) As in (b), it is clear that every simple arc $A$ in $S^{*}$ has as its inverse a topological polyhedron which contains no nontrivial two- 
cycle and so does not separate $S$. Consequently, $A$ does not separate $S^{*}$.

LEMMA 3. Let $K$ be a compact space and $f$ a continuous mapping of $K$ onto a simply-connected space $C$ so that each $x$ in $C$ has the same finite number $k$ of inverses under $f$ and so that each point $a$ in $K$ has a neighborhood which is mapped univalently by $f$. Then $K$ has $k$ components and each of these is mapped homeomorphically by $f$ onto $C$. (The definition of 'simply-connected' used here is that of [1].)

Proof. (In the proof it is assumed that $C$ satisfies the first axiom of countability. Only simple alterations are required to avoid this restriction.) It is first shown that each point $a$ in $K$ has a neighborhood which is mapped homeomorphically onto a neighborhood of $f a$. Let $a_{1}=a, \cdots, a_{k}$ be the points which map onto $f a$ by $f$. Let $N_{1}, \cdots, N_{k}$ be mutually disjoint compact neighborhoods of $a_{1}, \cdots, a_{k}$, respectively, which are mapped univalently by $f$. Suppose $f N_{1}$ is not a neighborhood of $f a$. Then there is a sequence $\left\{x_{n}\right\}$ of points in $C$ converging to $f a$ and having the property that $\left(f^{-1}\left(x_{n}\right)\right)$ $\cap N_{1}=\Lambda$ for all integers $n$. By using the compactness of $K, k$ convergent sequences $\left\{a_{i}^{1}\right\}, \cdots,\left\{a_{i}^{k}\right\}$ in $K$ can be found so that $a_{i}^{m} \neq a_{i}^{m^{\prime}}$ for all integers $i, m$, and $m^{\prime}$ satisfying $1 \leqq m<m^{\prime} \leqq k$ and so that $f\left(a_{i}^{m}\right)=x_{n_{i}}$, where $\left\{x_{n_{i}}\right\}$ is a subsequence of $\left\{x_{n}\right\}$. Let $b_{1}, \cdots, b_{k}$ be the limits of $\left\{a_{i}^{1}\right\}, \cdots,\left\{a_{i}^{\mathbf{k}}\right\}$, respectively. Clearly each $b_{m}$ is an $a_{m^{\prime}}$. Suppose $b_{m}=a_{i_{0}}$. Then almost all the terms of $\left\{a_{i}^{m}\right\}$ are in $N_{i_{0}}$ and so only finitely many terms of $\left\{a_{i}^{m^{\prime}}\right\}, m^{\prime} \neq m$, are in $N_{i_{0}}$. Thus $b_{m^{\prime}} \neq a_{i_{0}}$, if $m^{\prime} \neq i_{0}$. This shows that the $b_{m}$ are a permutation of the $a_{m^{\prime}}$. Say $a=a_{1}=b_{m_{0}}$. Thus infinitely many terms of $\left\{a_{i}^{m_{0}}\right\}$ are in $N_{1}$; that is, $f^{-1}\left(x_{n_{i}}\right) \cap N_{1} \neq \Lambda$ for infinitely many integers $i$. This contradiction shows that $f\left(N_{1}\right)$ is a neighborhood of $f a$. Since $N_{1}$ is compact and $f$ is univalent and continuous on $N_{1}, f$ is a homeomorphism of $N_{1}$ onto a neighborhood of $f a$. Since $C$ is locally-connected, $N_{1}$ can be restricted to be connected, in addition to having the other properties.

Let $K_{1}$ be any component of $K$ and let $a$ be any point of $K_{1}$. Then $a$ has a connected neighborhood $N$ which is mapped homeomorphically onto a neighborhood of $f a$, and since $K_{1}$ is a component, $N$ is a subset of $K_{1}$. Thus $f K_{1}$ is an open subset of $C$. Since $K_{1}$ is closed, it is compact and so $f K_{1}$ is also closed. So $f K_{1}=C$, since $C$ is connected. Thus $\left(K_{1}, f\right)$ is a covering space for $C$, and since $C$ is simply connected, $f$ is a homeomorphism of $K_{1}$ onto $C$ (see [1]).

It is now clear that $K$ has a finite number of components and that each of these is mapped homeomorphically by $f$ onto $C$.

Definition. Let ' $\boldsymbol{~}$ ' denote the subset of $R \times R$ consisting of the 
pairs $(u, v)$ for which $u>0, v>0, u^{2}+v^{2} \leqq 1$ and let ' $C$ ' hereafter denote the oriented arc of points $(u, v)$ for which $u \geqq 0, v \geqq 0, u^{2}+v^{2}=1$, from $(0,1)$ to $(1,0)$. A pseudosection $P$ at $x$ relative to $T$, where $x$ is any point on an exceptional orbit of $T$, its period being, say, $k$, is a homeomorphism of the closure $\bar{\Sigma}$ of $\Sigma$ into $S$ so that

(a) $P \bar{\Sigma}$ intersects no exceptional orbit except that containing $x$ and intersects that orbit only at $P(0,0)=x$;

(b) an orbit of $T$ which intersects $P \bar{\Sigma}$ intersects it exactly once;

(c) the orbit containing $P(0, u), 0<u \leqq 1$, intersects $P \bar{\Sigma}$ only at $P(0, u)$ and $P(u, 0)$; furthermore $P(u, 0)=T(n / k, P(0, u))$ for some integer $n$, independent of $u$, which satisfies $1 \leqq n \leqq k-1$ and is relatively prime to $k$.

The pair $(k, n)$ is called the characteristic pair of the pseudosection. The set $V$ of points of the form $T(t, P(u, v)),(t,(u, v)) \in R \times \bar{\Sigma}$, contains the orbit of $x$ in its interior. It is called the fiber-neighborhood generated by $P . V$ is a solid torus. Its boundary is the torus $R$ consisting of all points of the form $T(t, P(u, v)),(t,(u, v)) \in R \times C$.

Lemma 4. If $x$ is any point on an exceptional orbit of $T$, then there exists a pseudosection at $x$ relative to $T$.

Proof. Let $A$ be the orbit containing $x$. As in [2, p. 262], there exist a compact set $K$ in $S$ containing $x$ and a symmetric closed interval $I=[-\epsilon, \epsilon]$ in $R$ containing 0 in its interior so that the set $T(I, K)$ is a neighborhood of $x$ with the properties that each of its points is expressible as $T(r, y)$ for uniquely determined $r$ in $I$ and $y$ in $K$ and that $T(I, K)$ intersects no exceptional orbit except $A$. ( $T$ is then a homeomorphism of $I \times K$ onto $T(I, K)$.) Furthermore $K$ can be restricted so that, in addition to the properties above, except for $A$ each orbit which intersects $K$ intersects $K$ in exactly $k$ distinct points.

The image under $\Phi$ of $T(I, K)$ is a neighborhood in $S^{*}$ of $\Phi x=X$. Since $S^{*}$ is homeomorphic to $S^{2}$, there is a homeomorphism $h$ of the unit disc into this neighborhood under which the origin is mapped onto $X$. Throughout this proof ' $R_{r}$ ' denotes the set of all points $h(u, v)$ for all pairs $(u, v)$ of real numbers satisfying $u \geqq 0$ and $r \leqq u^{2}$ $+v^{2} \leqq 1$, ' $L_{r}$ ' the set of all such points for which $u \leqq 0$ and $r \leqq u^{2}+v^{2}$ $\leqq 1$, ' $U_{r}^{\prime}$ ' the set of all such points for which $u=0$ and $r \leqq v \leqq 1$, and ' $V_{r}$ ' the set of all such points for which $u=0$ and $-r \geqq v \geqq-1$.

Each point of $K-\{x\}$ has a neighborhood which is mapped univalently by $\Phi$. For if this is not the case there are sequences $\left\{z_{n}\right\}$ and $\left\{z_{n}^{\prime}\right\}$ in $K-\{x\}$ converging to a point $z$ in $K-\{x\}$ so that, for each integer $n, z_{n}$ and $z_{n}^{\prime}$ are distinct points on the same orbit. Now 
$z_{n}^{\prime}=T\left(t_{n}, z_{n}\right)$ for some $t_{n}$ with $\epsilon<t_{n}<1-\epsilon$. There is an increasing sequence $\left\{n_{i}\right\}$ of integers so that $\left\{t_{n_{i}}\right\}$ converges, say to $t_{0}$, with $0<t_{0}<1$. But then $z=\lim \left\{z_{n_{i}}^{\prime}\right\}=\lim \left\{T\left(t_{n_{i}}, z_{n_{i}}\right)\right\}=T\left(t_{0}, z\right)$, which contradicts the fact that $z$ has period 1 .

Thus for fixed $r$ with $0<r<1$, Lemma 3 can be applied to prove that $F_{r}=\Phi^{-1}\left(R_{r}\right)$ has $k$ components which are mapped homeomorphically onto $R_{r}$ by $\Phi$. Let $y$ be any inverse under $\Phi$ of $h(0,1)$ and let $P_{r}$ be the component of $F_{r}$ containing $y$. Clearly $P_{s} \subset P_{r}$ if $0<r \leqq s<1 . P_{0}=\left(\cup_{0<r<1} P_{r}\right) \cup\{x\}$ is a compact subset of $K$ which is mapped homeomorphically onto $R_{0}$ by $\Phi$. In a similar way a compact subset $Q_{0}$ of $K$ is constructed which contains $y$ and is mapped homeomorphically onto $L_{0}$ by $\Phi$. Also, similar arguments show that the inverse of $U_{0}$ (or $V_{0}$ ) consists of $k$ simple arcs having only $x$ in common. Let ' $W_{0}$ ' denote the part of $P_{0}$ mapping onto $U_{0}$ and ' $W_{0}^{\text {' }}$ the part of $Q_{0}$ mapping onto $U_{0}$. Then $W_{0}$ and $W_{0}^{\prime}$ are two of the simple arcs referred to above and since they have $y$ in common they are the same. It will now be shown that $P_{0}$ and $Q_{0}$ have only $W_{0}$ in common, so that their union is almost a pseudosection.

The part of $P_{0}$ mapping onto $V_{0}$ and the corresponding part of $Q_{0}$ either have only $x$ in common or coincide. In the latter case $P_{0} \cup Q_{0}$ is mapped homeomorphically onto $R_{0} \cup L_{0}$ by $\Phi$, and so is homeomorphic to a disc with $x$ as an interior point. Since $T\left(I, P_{0} \cup Q_{0}\right)$ is homeomorphic to $I \times\left(P_{0} \cup Q_{0}\right)$, by the Brouwer theorem $x$ is an interior point of $T\left(I, P_{0} \cup Q_{0}\right)$. But this is a contradiction. For orbits which traverse $T\left(I, P_{0} \cup Q_{0}\right)$ traverse it exactly once, whereas all except $A$ must traverse it $k$ times since $x$ has period $1 / k$. Thus $P_{0}$ and $Q_{0}$ have only $W_{0}$ in common. Clearly there is a homeomorphism $P^{\prime}$ of $\bar{\Sigma}$ onto $P_{0} \cup Q_{0}$ having all the properties of a pseudosection except possibly the last part of condition (c).

There is a uniquely determined function $g$ of $] 0,1]$ to $] \epsilon, 1-\epsilon[$ satisfying

$$
P^{\prime}(u, 0)=T\left(g u, P^{\prime}(0, u)\right),
$$

for all $u$ in $] 0,1] . g$ is continuous and can be extended to be continuous and satisfy (1) on $[0,1]$. Since $x=P^{\prime}(0,0)=T\left(g 0, P^{\prime}(0,0)\right)=T(g 0, x)$, it follows that $g 0=n / k$ for some integer $n$ satisfying $1 \leqq n \leqq k-1$. Let $f$ be any continuous function from $\bar{\Sigma}$ to $R$ satisfying $f(0, u)=0$ and $f(u, 0)=(n / k)-g u$, for $0 \leqq u \leqq 1 . f$ can be chosen so that the maximum value of $|f|$ in $\bar{\Sigma}$ is less than 1 . Define $P$ from $\bar{\Sigma}$ to $S$ by

$$
P(u, v)=T\left(f(u, v), P^{\prime}(u, v)\right),
$$

for all $(u, v)$ in $\bar{\Sigma}$. 
The proof will be completed by showing that $n$ and $k$ are relatively prime, for it is easy to verify that $P$ has all the remaining properties of a pseudosection. Let ' $m$ ' denote the greatest common divisor of $n$ and $k$. Choose $c$ and $d$ so that $n=c m$ and $k=d m$. For any integer $i$, let ' $K_{i}$ ' denote $T(i / k, P \bar{\Sigma})$. Then the sets $K_{0}, K_{n}, K_{2 n}, \cdots, K_{(d-1) n}$ fit together to form a two-cell having $x$ as an interior point. This two-cell can be used to construct a neighborhood of $x$ in $S$ (compare the construction of the neighborhood $T\left(I, P_{0} \cup Q_{0}\right)$ in the next to the preceding paragraph) and since all orbits, except $A$, which intersect the two-cell intersect it $d$ times, it is seen that $x$ has period $1 / d$. Thus $d=k, m=1$, and so $n$ and $k$ are relatively prime. [3].

Lemma 5. The orbits of $T$ constitute a fibering of $S$ in the sense of

Proor. Since there is a true section through any point of an ordinary orbit [2, p. 221], it is clear that each ordinary orbit has a fiberneighborhood of the required type. It was shown in Lemma 4 that there is a pseudosection at any point of an exceptional orbit, and this generates a fiber-neighborhood of the exceptional orbit of the required type.

REMARK. In view of Lemma 5, $\$ 11$ of [3] can be applied. There are then two uniquely determined positive relatively prime integers $k^{\prime}$ and $k^{\prime \prime}$, with $k^{\prime}<k^{\prime \prime}$, and a homeomorphism $\psi$ of $S$ onto $S$ mapping the orbits of $\mathrm{II}_{\left(k^{\prime}, k^{\prime \prime}\right)}$ onto those of $T$. However, it is not necessarily true that $T(t, \psi x)=\psi\left(\Pi_{\left(k^{\prime}, k^{\prime \prime}\right)}(t, x)\right)$ for all $(t, x)$ in $\boldsymbol{R} \times S$. Some further adjustment of $\psi$ may be necessary. In proving the lemmas necessary to effect this adjustment, it turns out that there is at hand most of the machinery for proving the results of [3] that are used here. Thus it appears advisable to include proofs of these results also.

Lemma 6. Let $T^{\prime}$ and $T^{\prime \prime}$ be any two functions satisfying the conditions imposed on $T$ in the hypothesis of the theorem. Suppose further that $x^{\prime}$ and $x^{\prime \prime}$ are on exceptional orbits of $T^{\prime}$ and $T^{\prime \prime}$ and that $P^{\prime}$ and $P^{\prime \prime}$ are pseudosections at $x^{\prime}$ and $x^{\prime \prime}$ relative to $T^{\prime}$ and $T^{\prime \prime}$, respectively, both with the same characteristic pair $(k, n)$. Then there is a homeomorphism $\psi$ of the fiber-neighborhood $V^{\prime}$ generated by $P^{\prime}$ onto that, $V^{\prime \prime}$, generated by $P^{\prime \prime}$ so that $\psi x^{\prime}=x^{\prime \prime}$ and so that furthermore $\psi\left(T^{\prime}(t, y)\right)=T^{\prime \prime}(t, \psi y)$ for all $(t, y)$ in $R \times S$.

Proof. Given $y$ in $V^{\prime}$, choose $(t, \quad(u, v)) \in R \times \bar{\Sigma}$ so that $y=T^{\prime}\left(t, P^{\prime}(u, v)\right)$. Then define $\psi y$ to be $T^{\prime \prime}\left(t, P^{\prime \prime}(u, v)\right)$. Although $(t,(u, v))$ is not uniquely determined by $y$, it can be seen that, since $P^{\prime}$ and $P^{\prime \prime}$ have the same characteristic pair, $\psi y$ is well defined. It is 
readily verified that $\psi$ has the required properties.

REMARKs. The two-cell $K_{0} \cup K_{n} \cup \ldots \cup K_{(k-1) n}$ involved in the proof of Lemma 4 has as its boundary the oriented curve $M=E_{0}$ $\cup E_{n} \cup \ldots \cup E_{(k-1) n}$, where, for any integer $i, E_{i}$ is $T(i / k, P C)$. Thus $M$ is homotopic to zero in $V$. The oriented curve $D$ consisting of the points of the form $T(g t, P(\sin t, \cos t)), 0 \leqq t \leqq \pi / 2$, where $g$ is some fixed monotonically decreasing function from $[0, \pi / 2]$ on to $[0,-n / k]$, lies on the surface $R$ of $V$ and intersects each orbit in $R$ exactly once. If $H$ is the (oriented) orbit through $P(0,1)$, then the homotopy classes of $H$ and $D$ form a basis for the fundamental group (B) of $R$ with base point $P(0,1)$.

Lemma 7. Let $x$ be any point on an exceptional orbit of $T$ and let $P$ be a pseudosection at $x$ relative to $T$ with characteristic pair $(k, n)$. Then using the notation established in the preceding paragraph, $M \sim D^{k} \cdot H^{n}$.

Proof. (In the proof ' $(a, b)$ ' denotes the oriented arc $T(t, P(0,1))$, the parameter varying from $a$ to $b$.) The function $\Omega$ defined on the rectangle $[0,1] \times[0, \pi / 2]$ by

$$
\Omega(u, t)=T(u \cdot g t, P(\sin t, \cos t))
$$

for $(u, t) \in[0,1] \times[0, \pi / 2]$, shows that $E_{0} \cdot(n / k, 0) \sim D$. In a similar way, the following homotopies are established.

$$
\begin{aligned}
E_{n} & \sim(n / k, 0) \cdot E_{0} \cdot(n / k, 2 n / k), \\
E_{2 n} & \sim(2 n / k, 0) \cdot E_{0} \cdot(n / k, 3 n / k), \\
. & \cdot \cdot \cdot \cdot . \cdot . \cdot . \cdot . \cdot . \cdot \cdot, \\
E_{(k-1) n} & \sim((k-1) n / k, 0) \cdot E_{0} \cdot(n / k, k n / k) .
\end{aligned}
$$

Thus

$$
\begin{aligned}
M & \sim E_{0} \cdot(n / k, 0) \cdot E_{0} \cdot(n / k, 2 n / k) \cdot(2 n / k, 0) \cdots \cdot E_{0} \cdot(n / k, k n / k) \\
& \sim\left[E_{0} \cdot(n / k, 0)\right]^{k} \cdot(0, n) \sim D^{k} \cdot H^{n} .
\end{aligned}
$$

Lemma 8. Under the hypothesis of Lemma 7, if $A$ is a closed curve in $R$, then $A$ is homotopic to zero in $V$ if and only if $A$ is homotopic to $M^{a}$, for some integer $a$.

Proof. Clearly $M^{a}$ is homotopic to zero in $V$, since $M$ is. Conversely, since the subgroup of (s) containing the homotopy classes of curves homotopic to zero in $V$ is an infinite cyclic group containing the class of $M$, it suffices to show that $M$ is not homotopic to a curve of the form $N^{b}$ for some closed curve $N$ and some integer $b>1$. That no such $N$ and $b$ exist follows from the fact that $M=D^{k} \cdot H^{n}$ and that $\boldsymbol{n}$ and $k$ are relatively prime. 
Lemma 9. Let $\Gamma_{1}, \cdots, \Gamma_{8}$ be disjoint open discs in $S^{*}$ whose union includes all exceptional points. Then there exists a compact set $\Delta$ in $S$ which intersects each orbit of $\Phi^{-1}\left(S^{*}-\Gamma_{1}-\cdots-\Gamma_{s}\right)$ exactly once; that is, $\Delta$ is a true section of $\Phi^{-1}\left(S^{*}-\Gamma_{1}-\cdots-\Gamma_{s}\right) . \Phi$ is then a homeomorphism of $\Delta$ onto $S^{*}-\Gamma_{1}-\cdots-\Gamma_{8}$.

PRoof. If $s=1, S^{*}-\Gamma_{1}$ is a two-cell, and by the preliminary remarks preceding Lemma $1, \Delta$ is seen to exist.

If $s>1, U=S^{*}-\Gamma_{1}-\cdots-\Gamma_{s}$ is homeomorphic to a disc with $s-1$ holes. $U$ can be divided into $s$ two-cells, $S_{1}, S_{2}, \cdots, S_{s}$, as indicated in the accompanying figure for $s=4$. Each of the sets $\Phi^{-1}\left(S_{i}\right)$ has a cross-section $\Delta_{i}$, and it is clear that the $\Delta_{i}$ can be adjusted so as to fit together to form the required $\Delta$.

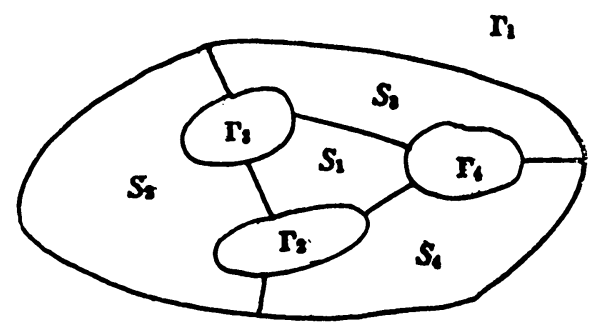

LemMa 10. T has at most two exceptional orbits.

Proof. (In this proof ' $V{ }_{i}^{\prime}$ ' ' $R_{i}$ ', ' $M_{i}$ ', $\cdots$ are used with reference to the pseudosection $P_{i}$ in the same way that ' $V$ ', ' $R$ ', ' $M$ ', . . were used with reference to $P$, earlier. The function $\tilde{P}_{i}$ defined on $\bar{\Sigma}$ by $\widetilde{P}_{i}(u, v)=P_{i}(v, u)$, for $(u, v)$ in $\bar{\Sigma}$, is the reorientation of the pseudosection $P_{i}$.)

Suppose, on the contrary, that $T$ has $s,>2$, exceptional orbits. Choose points $x_{1}, \cdots, x_{\varepsilon}$ on these orbits. Choose pseudosections $P_{1}, \cdots, P_{s}$ at $x_{1}, \cdots, x_{s}$, respectively, relative to $T$, so that no two of the fiber-neighborhoods generated thereby intersect. Let $\Gamma_{i}$ be the interior of $\Phi\left(V_{i}\right)$, and let $\gamma_{i}$ be the oriented image of the oriented curve $D_{i}, i=1, \cdots, s . \gamma_{i}$ is the boundary of $\Gamma_{i}, i=1, \cdots, s$. Choose a cross-section $\Delta$ of $X=\Phi^{-1}\left(S^{*}-\Gamma_{1}-\cdots-\Gamma_{s}\right)$.

Upon reorienting certain ones of the $\gamma_{i}$ (i.e., reorienting certain $\left.P_{i}\right)$, if necessary, the fundamental group of $X$, which is homeomorphic to the direct product of a circle and $\Delta$, is seen to be isomorphic to the free group with generators $\gamma_{1}, \cdots, \gamma_{s}, H$, and relations $\gamma_{1} \cdots \cdots \gamma_{s}=1, \gamma_{1} \cdot H=H \cdot \gamma_{1}, \cdots, \gamma_{s} \cdot H=H \cdot \gamma_{s}$. Since $\gamma_{i}$ is homotopic in $R_{i}$ to $D_{i}^{k_{i}} \cdot H^{a_{i}}$ for a suitable integer $a_{i}, i=1, \cdots, s$, the fundamental group of $S$ is seen to be isomorphic to the free group 
with generators $\gamma_{1}, \cdots, \gamma_{s}, H$ and relations $\gamma_{1} \cdots \gamma_{s}=1, \gamma_{1} \cdot H$ $=H \cdot \gamma_{1}, \cdots, \gamma_{s} \cdot H=H \cdot \gamma_{s}, \gamma_{1}^{k} \cdot H^{-a_{1} k_{1}+n_{1}}=1, \cdots, \gamma^{k_{s}} \cdot H^{-a_{s} k_{s}+n_{s}}=1$, where $\left(k_{i}, n_{i}\right)$ is the characteristic pair of $P_{i}, i=1, \cdots, s$. By adjoining the relation $H=1$, a factor group isomorphic to the free group with generators $\gamma_{1}, \cdots, \gamma_{8}$ and relations $\gamma_{1}^{k_{1}}=\cdots=\gamma_{s}^{k_{k}}=1$ is obtained. Since none of the $k_{i}$ is 1 and since the $k_{i}$ are relatively prime in pairs this factor group is not a trivial group. This is a contradiction, since the fundamental group of $S$ is trivial.

REMARKs. Suppose that $T$ has two exceptional orbits with periods $k^{\prime}$ and $k^{\prime \prime}$. Choose points $x^{\prime}$ and $x^{\prime \prime}$ on these orbits and choose pseudosections $P^{\prime}$ and $P^{\prime \prime}$ at $x^{\prime}$ and $x^{\prime \prime}$, respectively, relative to $T$, so that the two fiber-neighborhoods generated thereby do not intersect. Choose orientations of $P^{\prime}$ and $P^{\prime \prime}$ so that the oriented images $\gamma^{\prime}$ and $\gamma^{\prime \prime}$ of the oriented curves $D^{\prime}$ and $D^{\prime \prime}$ are homotopic in $S^{*}-\Gamma^{\prime}-\Gamma^{\prime \prime}$, where $\Gamma^{\prime}$ and $\Gamma^{\prime \prime}$ are the interiors of $\Phi\left(V^{\prime}\right)$ and $\Phi\left(V^{\prime \prime}\right)$. As in the proof of Lemma 10 , it is seen that the fundamental group of $S$ is isomorphic to the free group with generators $\gamma^{\prime}$ and $\gamma^{\prime \prime}$ and relations

$$
\begin{gathered}
\gamma^{\prime}=\gamma^{\prime \prime}, \quad \gamma^{\prime} \cdot H=H \cdot \gamma^{\prime}, \quad \gamma^{\prime \prime} \cdot H=H \cdot \gamma^{\prime \prime}, \quad \gamma^{\prime k^{\prime}} \cdot H^{-a^{\prime} k^{\prime}+n^{\prime}}=1, \\
\gamma^{\prime \prime k^{\prime \prime}} \cdot H^{-a^{\prime \prime} k^{\prime \prime}+n^{\prime \prime}}=1,
\end{gathered}
$$

where $\left(k^{\prime}, n^{\prime}\right)$ and $\left(k^{\prime \prime}, n^{\prime \prime}\right)$ are the characteristic pairs of $P^{\prime}$ and $P^{\prime \prime}$ and $a^{\prime}$ and $a^{\prime \prime}$ are suitable integers. This group is a trivial group if and only if it can be deduced that $\gamma^{\prime}=\gamma^{\prime \prime}=H=1$. This is the case if and only if the system of equations

$$
\begin{aligned}
x k^{\prime}+y k^{\prime \prime} & =1, \\
x\left(-a^{\prime} k^{\prime}+n^{\prime}\right)+y\left(-a^{\prime \prime} k^{\prime \prime}+n^{\prime \prime}\right) & =0, \\
u k^{\prime}+v k^{\prime \prime} & =0, \\
u\left(-a^{\prime} k^{\prime}+n^{\prime}\right)+v\left(-a^{\prime \prime} k^{\prime \prime}+n^{\prime \prime}\right) & =1
\end{aligned}
$$

has integral solutions $x, y, u, v$. This is the case if and only if

$$
\left(\begin{array}{cc}
k^{\prime} & k^{\prime \prime} \\
-a^{\prime} k^{\prime}+n^{\prime} & -a^{\prime \prime} k^{\prime \prime}+n^{\prime \prime}
\end{array}\right) \cdot\left(\begin{array}{ll}
x & u \\
y & v
\end{array}\right)=\left(\begin{array}{ll}
1 & 0 \\
0 & 1
\end{array}\right),
$$

that is, if and only if the left matric factor is unimodular, or

$$
k^{\prime}\left(-a^{\prime \prime} k^{\prime \prime}+n^{\prime \prime}\right)-k^{\prime \prime}\left(-a^{\prime} k^{\prime}+n^{\prime}\right)= \pm 1
$$

In case the negative sign is appropriate, the equation (2) can be rewritten

$$
k^{\prime}\left(\left(a^{\prime \prime}-1\right) k^{\prime \prime}+\left(k^{\prime \prime}-n^{\prime \prime}\right)\right)-k^{\prime \prime}\left(\left(a^{\prime}-1\right) k^{\prime}+\left(k^{\prime}-n^{\prime}\right)\right)=1 .
$$


Now it is easily seen that if $P$ has the characteristic pair $(k, n)$, then $\widetilde{P}$ has the pair $(k, k-n)$. Thus, by suitably orienting $P^{\prime}$ and $P^{\prime \prime}$, it is possible to achieve the positive sign in (2). When such orientations have been chosen, the pair $\left(P^{\prime}, P^{\prime \prime}\right)$ is said to be canonically oriented.

An equation of the form $A k^{\prime} k^{\prime \prime}+k^{\prime} n^{\prime \prime}-k^{\prime \prime} n^{\prime}=1$ has a unique solution for $A, n^{\prime}, n^{\prime \prime}$, corresponding to given, relatively prime integers $k^{\prime}$ and $k^{\prime \prime}$, both larger than 2, subject to the conditions $0<n^{\prime}<k^{\prime}$, $0<n^{\prime \prime}<k^{\prime \prime}$. In particular, $A=0$. Also, since (2) has this form, it is seen that for canonically oriented pseudosections, the characteristic pairs are determined by the periods.

Let $\Delta$ be a cross-section of the orbits of $\Phi^{-1}\left(S^{*}-\Gamma^{\prime}-\Gamma^{\prime \prime}\right)$ and let $\kappa^{\prime}$ and $\kappa^{\prime \prime}$ be the oriented intersections of $\Delta$ with $R^{\prime}$ and $R^{\prime \prime}$, respectively; that is, oriented so that $\Phi$ is an orientation preserving homeomorphism of $\kappa^{\prime}$ and $\kappa^{\prime \prime}$ on to $\gamma^{\prime}$ and $\gamma^{\prime \prime}$, respectively. $\Delta$ can be adjusted so that $\kappa^{\prime}$ and $D^{\prime}$ are homotopic in $R^{\prime}$. Thus, $M^{\prime} \sim \kappa^{\prime k^{\prime}} \cdot H^{n^{\prime}}$. Also, $M^{\prime \prime} \sim K^{\prime \prime k^{\prime \prime}} \cdot H^{c k^{\prime \prime}+n^{\prime \prime}}$ for some integer $c$. The condition $A=0$ of the preceding paragraph here yields $c=0$. Thus also $\kappa^{\prime \prime}$ and $D^{\prime \prime}$ are homotopic in $R^{\prime \prime}$. A choice of $\Delta$ with this property will be called a "special" choice, subsequently.

Proof of THE THEOREM. The proof will be given only for the case that $T$ has two exceptional orbits. The proof for the remaining cases can be made by using one (or two) ordinary orbits in place of one (or two) of the exceptional orbits. Suppose the exceptional orbits of $T$ have periods $k^{\prime}$ and $k^{\prime \prime}$, with $k^{\prime}<k^{\prime \prime}$. Choose points $x^{\prime}$ and $x^{\prime \prime}$ on these orbits ( $x^{\prime}$ has period $k^{\prime}$ ), and choose canonically oriented pseudosections $P^{\prime}$ and $P^{\prime \prime}$ at $x^{\prime}$ and $x^{\prime \prime}$, respectively, relative to $T$, so that the two fiber-neighborhoods $V^{\prime}$ and $V^{\prime \prime}$ generated thereby do not intersect. Choose a special cross-section $\Delta$ of $\Phi^{-1}\left(S^{*}-\Gamma^{\prime}-\Gamma^{\prime \prime}\right)$, where $\Gamma^{\prime}$ and $\Gamma^{\prime \prime}$ are the interiors of $\Phi\left(V^{\prime}\right)$ and $\Phi\left(V^{\prime \prime}\right)$. Similarly, choose $\hat{x}^{\prime}, \hat{x}^{\prime \prime}, \widehat{P}^{\prime}, \widehat{P}^{\prime \prime}, \hat{V}^{\prime}, \widehat{V}^{\prime \prime}, \widehat{\Delta}$, relatively to $\Pi=\Pi_{\left(k^{\prime}, k^{\prime \prime}\right)}$, where $\hat{x}^{\prime}$ has period $k^{\prime}$. In view of the preceding remark, the characteristic pairs of $P^{\prime}$ and $\widehat{P}^{\prime}$, as well as those of $P^{\prime \prime}$ and $\widehat{P}^{\prime \prime}$, are the same. Thus by Lemma 6 there is a homeomorphism $\psi$ of $\hat{V}^{\prime} \cup V^{\prime \prime}$ onto $V^{\prime} \cup V^{\prime \prime}$ so that

$$
\psi(\Pi(t, y))=T(t, \psi y), \quad(t, y) \in R \times\left(\widehat{V}^{\prime} \cup \widehat{V}^{\prime \prime}\right) .
$$

It now remains to extend $\psi$ to $\Phi^{-1}\left(S^{*}-\Gamma^{\prime}-\Gamma^{\prime \prime}\right)$. Since $\Delta$ and $\hat{\Delta}$ are special it is clear that $\psi \bar{\kappa}^{\prime}$ is homotopic to $\kappa^{\prime}$ in $R^{\prime}$ and $\psi \widehat{\kappa}^{\prime \prime}$ is homotopic to $\kappa^{\prime \prime}$ in $R^{\prime \prime}$, and so $\Delta$ can be adjusted so that $\psi \bar{\kappa}^{\prime}=\kappa^{\prime}$ and $\psi \hat{\kappa}^{\prime \prime}=\kappa^{\prime \prime}$. The extension of $\psi$ is now readily made. 


\section{BIBLIOGRAPHY}

1. C. Chevalley, Theory of Lie groups, Princeton University Press, 1946.

2. D. Montgomery and L. Zippin, Topological transformation groups, Interscience Press, 1955.

3. H. Seifert, Topologie dreidimensionaler gefaserter Räume, Acta Math. vol. 60 (1932) pp. 147-238.

4. G. T. Whyburn, Analytic topology, Amer. Math. Soc. Colloquium Publications, vol. $28,1942$.

RUTGERS UNIVERSITY

\section{A NOTE ON RECURRENT FLOWS}

CHARLES DAVID GORMAN

Let $R$ be a metric space and let $f: R \times\{t \mid t$ is real $\} \rightarrow R$ be a continuous flow in $R$. The flow $f$ is said to be recurrent (in the sense of Gottschalk and Hedlund) provided that if $\epsilon$ and $s$ are positive real numbers, then there exists a real number $t$ such that $t>s$ and $\rho(x, f(x, t))$ $<\epsilon$ for all $x \in R$.

THEOREM. Consider the statements:

(I) $f$ is recurrent,

(II) There exists a sequence $t_{1}<t_{2}<\cdots \rightarrow+\infty$ of real numbers such that $\lim _{n \rightarrow \infty} f\left(A, t_{n}\right)=A$ for every closed subset $A$ of $R$,

(III) There exists a sequence $t_{1}<t_{2}<\cdots \rightarrow+\infty$ of real numbers such that $\lim \sup _{n \rightarrow \infty} f\left(A, t_{n}\right) \subset A$ for every closed subset $A$ of $R$.

Then,

(1) I implies II; II implies III,

(2) If $R$ is compact, then I, II, III are equivalent.

Proof. Assume I. There exists a sequence $t_{1}<t_{2}<\cdots \rightarrow+\infty$ of real numbers such that $\rho\left(x, f\left(x, t_{n}\right)\right)<1 / n$ for all $x \in R$ and all positive integers $n$. Let $A$ be a closed subset of $R$. If $x \in A$, then $f\left(x, t_{n}\right) \rightarrow x$. Hence $A \subset \lim \inf _{n \rightarrow \infty} f\left(A, t_{n}\right)$. Suppose $x \in \lim \sup _{n \rightarrow \infty} f\left(A, t_{n}\right)$. There exist $x_{1}, x_{2}, \cdots \in A$ and a sequence $n_{1}<n_{2}<\cdots \rightarrow+\infty$ of positive integers such that $\rho\left(x, f\left(x_{i}, t_{n_{i}}\right)\right) \rightarrow 0$. Since $\rho\left(x_{i}, f\left(x_{i}, t_{n_{i}}\right)\right)<1 / n_{i}$ for all positive integers $i$, it follows that $\rho\left(x_{i}, f\left(x_{i}, t_{n_{i}}\right)\right) \rightarrow 0$ whence $x_{i} \rightarrow x$ and $x \in A$. Hence $\lim \sup _{n \rightarrow \infty} f\left(A, t_{n}\right) \subset A$. This proves that I implies II.

Presented to the Society, September 2, 1955 ; received by the editors January 5 , 1955 and, in revised form, on April 18, 1955. 